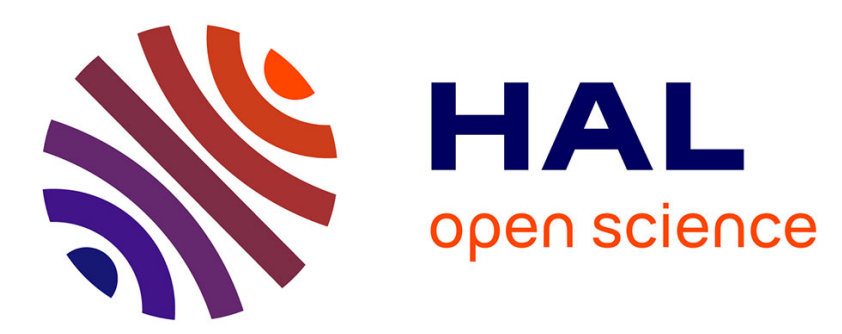

\title{
Focalisation à travers une interface plane: comparaison entre la méthode de retournement temporel et la technique des surfaces de Fermat
}

D. Cassereau, M. Fink

\section{- To cite this version:}

D. Cassereau, M. Fink. Focalisation à travers une interface plane: comparaison entre la méthode de retournement temporel et la technique des surfaces de Fermat. Journal de Physique IV Proceedings, 1994, 04 (C5), pp.C5-893-C5-896. 10.1051/jp4:19945195 • jpa-00252878

\section{HAL Id: jpa-00252878 \\ https://hal.science/jpa-00252878}

Submitted on 1 Jan 1994

HAL is a multi-disciplinary open access archive for the deposit and dissemination of scientific research documents, whether they are published or not. The documents may come from teaching and research institutions in France or abroad, or from public or private research centers.
L'archive ouverte pluridisciplinaire HAL, est destinée au dépôt et à la diffusion de documents scientifiques de niveau recherche, publiés ou non, émanant des établissements d'enseignement et de recherche français ou étrangers, des laboratoires publics ou privés. 


\title{
Focalisation à travers une interface plane : comparaison entre la méthode de retournement temporel et la technique des surfaces de Fermat
}

\author{
D. CASSEREAU et M. FINK
}

Laboratoire Ondes et Acoustique, Université Paris VII, E.S.P.C.I., 10 rue Vauquelin, 75231 Paris cedex 05, France

\begin{abstract}
Résumé - Dans cet article, nous étudions la focalisation d'un champ de pression acoustique à travers une interface plane séparant deux fluides. Nous comparons les résultats obtenus en utilisant le principe des surfaces de Fermat (dans ce cas, le problème se réduit à une simple loi de retard) avec ceux obtenus par. retournement temporel [1-5] (on tient compte des distortions créées par l'interface). Nous montrons ainsi que le retournement temporel génère un lobe principal légèrement plus large que la surface de Fermat, mais présente un niveau de lobe secondaire plus bas, ce qui peut être d'une importance fondamentale, par exemple pour les applications en imagerie ultrasonore.
\end{abstract}

Abstract - In this paper, we are interested in focusing an acoustic pressure field through a plane interface separating two fluids. We compare the results obtained using the Fermat's surface technique (in this case, the problem reduces to a time delay law) with those obtained by time-reversal [1-5] (we take into account the distortions generated by the interface). Thus, we show that the time-reversal method generates a focal pattern with a broader main lobe than using Fermat's surface technique, but also with a smaller side lobe level. This result is very important, particularly in the frame of ultrasonic imaging.

\section{1) Principe de la focalisation par retournement temporel}

Dans tout ce qui suit, nous considérons deux milieux fluides homogènes (de vitesses et densités $c_{1}$, $\rho_{1}, c_{2}$ et $\left.\rho_{2}\right)$ semi-infinis séparés par une interface plane située en $z=h>0$. On note $f_{x}$ et $f_{y}$ les fréquences spatiales duales des coordonnées d'espace $x$ et $y, f$ la fréquence duale du temps $t$.

L'apprentissage de la focalisation par retournement temporel est un processus en deux temps.

Dans un premier temps, on considère une source active, située à l'origine des coordonnées spatiales, qui génère un champ de pression $p_{i}(x, y, z, t)$ que l'on peut écrire sous la forme

$$
p_{i}(x, y, z, t)=g_{2}(x, y, z, t) \frac{*}{t} \varphi(t)=\frac{1}{4 \pi R} \delta\left(t-\frac{R}{c_{2}}\right) \frac{*}{t} \varphi(t),
$$

avec $R=\sqrt{x^{2}+y^{2}+z^{2}} \cdot g_{2}(x, y, z, t)$ est la fonction de Green d'espace libre du milieu 2 et $\varphi(t)$ une fonction quelconque du temps qui décrit les fluctuations temporelles de l'excitation de la source.

Pour écrire les conditions aux limites, nous calculons la transformée de Fourier de $p_{i}(x, y, z, t)$ par rapport à $x, y$ et $t$. Nous obtenons ainsi le champ incident dans l'espace dual pour $0<z<h$ sous la forme [6]

$$
\tilde{P}_{i}\left(f_{x}, f_{y}, z, f\right)=\tilde{\varphi}(f) \tilde{A}_{i}\left(f_{x}, f_{y}, f\right) \exp \left(j \nu_{2} z\right),
$$

où $\nu_{2}$ est une grandeur complexe définie par $\nu_{2}^{2}=f^{2} / c_{2}^{2}-f_{x}^{2}-f_{y}^{2}, \operatorname{Im}\left(\nu_{2}\right) \geq 0 . \tilde{A}_{i}\left(f_{x}, f_{y}, f\right)=$ $j /\left(2 \nu_{2}\right)$ résulte de la décomposition en spectre angulaire de la fonction de Green.

A l'interface entre les deux fluides, on crée un champ réfléchi et un champ transmis. Les conditions aux limites à satisfaire sont la continuité de la pression et du déplacement normal. On obtient 
alors un système linéaire de deux équations, où les inconnues sont les amplitudes des composantes réfléchie et transmise. On obtient ainsi le champ transmis sous la forme $[6,7]$

$$
\tilde{P}_{t}\left(f_{x}, f_{y}, z, f\right)=\tilde{T}_{2 \rightarrow 1}\left(f_{x}, f_{y}, f\right) \tilde{\varphi}(f) \tilde{A}_{i}\left(f_{x}, f_{y}, f\right) \exp \left(j \nu_{1} z\right)
$$

où $\nu_{1}$ est défini comme $\nu_{2}$, en remplaçant $c_{2}$ par $c_{1}$. Dans cette équation, $\tilde{T}_{2 \rightarrow 1}\left(f_{x}, f_{y}, f\right)$ est le coefficient de transmission du fluide 2 vers le fluide 1 .

Le miroir à retournement temporel est situé dans le fluide 1 en $z=z_{0}>h$. On suppose que chaque point de la surface du miroir est capable de mesurer le champ de pression et/ou sa dérivée normale [5]. Partant de (3), une transformée de Fourier inverse sur $f_{x}$ et $f_{y}$ permet d'obtenir le champ de pression en tout point sur le miroir. Un calcul itératif sur les fréquences $f$ permet d'obtenir ce même champ en régime transitoire.

Au cours de la seconde étape du processus, la source active est retirée et le miroir fonctionne en mode émission. Nous ne considérons ici que le cas d'un miroir monté dans un baffle infiniment rigide (on peut faire des calculs similaires pour un baffle mou ou dans le cas de l'espace libre). Il en résulte que le champ diffracté $p_{d}(x, y, z, t)$ ne dépend que de la distribution de vitesse normale $\sigma_{0}\left(x_{s}, y_{s}, t\right)$ sur la surface du miroir [8-11]

$$
p_{d}(x, y, z, t)=2 \int_{S} g_{1}\left(x-x_{s}, y-y_{s}, z-z_{0}, t\right) \frac{*}{t} \sigma_{0}\left(x_{s}, y_{s}, t\right) d x_{s} d y_{s},
$$

où $S$ décrit la surface active du miroir et $g_{1}(x, y, z, t)$ est la fonction de Green d'espace libre du milieu 1. Si l'on introduit la fonction d'ouverture du miroir dans la définition de $\sigma_{0}\left(x_{s}, y_{s}, t\right)$, l'intégrale de (4) peut être étendue à tout le plan $z=z_{0}$; elle peut alors s'interpréter comme une convolution sur $x, y$ et $t[8]$. Dans l'espace dual, on a alors

$$
\tilde{P}_{d}\left(f_{x}, f_{y}, z, f\right)=2 \tilde{\Sigma}_{0}\left(f_{x}, f_{y}, f\right) \tilde{B}_{i}\left(f_{x}, f_{y}, f\right) \exp \left(-j \nu_{1} z\right)
$$

avec $\tilde{B}_{i}\left(f_{x}, f_{y}, f\right)=j /\left(2 \nu_{1}\right) \exp \left(j \nu_{1} z_{0}\right)$.

De même que précédemment, on écrit les conditions aux limites à l'interface pour obtenir le champ transmis au voisinage de la source initiale

$$
\tilde{P}_{t r}\left(f_{x}, f_{y}, z, f\right)=2 \tilde{T}_{1 \rightarrow 2}\left(f_{x}, f_{y}, f\right) \tilde{\Sigma}_{0}\left(f_{x}, f_{y}, f\right) \tilde{B}_{i}\left(f_{x}, f_{y}, f\right) \exp \left(-j \nu_{2} z\right),
$$

où $\tilde{T}_{1 \rightarrow 2}\left(f_{x}, f_{y}, f\right)$ est cette fois le coefficient de transmission du fluide 1 vers le fluide 2 .

Dans l'expérience de retournement temporel, la distribution de vitesse normale appliquée (phase 2) est égale au retourné temporel de la distribution de vitesse normale mesurée (phase 1). Il en résulte l'expression suivante $[5,8]$ :

$$
\tilde{\Sigma}_{0}\left(f_{x}, f_{y}, f\right)=-\frac{1}{2 j \pi f \rho_{1}}\left(\left.\frac{\partial}{\partial z} \tilde{P}_{t}\left(f_{x}, f_{y}, z, f\right)\right|_{z=z_{0}}\right)^{*} .
$$

Les deux étapes du processus sont illustrées par les figures 1 et 2 .

\section{2) Principe de la focalisation par surface de Fermat}

La méthode des surfaces de Fermat fait partie des méthodes classiques utilisées pour focaliser à travers une interface séparant deux fluides homogènes. On peut envisager deux approches similaires [3] :

* la géométrie du transducteur est telle que le temps de vol entre chaque point d'émission et le point focal (calculé le long d'un trajet géométrique satisfaisant la loi de Snell-Descartes) est constant,

* le transducteur est plan, mais on cherche une loi de retard à l'émission qui compense les écarts de temps de vol entre chaque point source et le point focal (les signaux issus des différents points de l'émetteur arrivent en même temps à la focale).

Ces deux approches sont similaires, même si la première est plus efficace et plus facile à réaliser expérimentalement. Pour nos calculs numériques, nous avons préféré mettre en cuvre la seconde approche ; notre démarche est illustrée par la figure 3.

L'inconvénient majeur de cette technique est qu'elle dépend de la géométrie de l'interface et de la position du point focal, alors que le retournement temporel est adaptatif [3].

\section{3) Résultats numériques}


Pour les simulations numériques, nous avons adopté les valeurs suivantes :

* la fréquence de travail est $f=1 \mathrm{MHz}$,

* les paramètres acoustiques sont $c_{1}=1500 \mathrm{~m} / \mathrm{s}, c_{2}=6000 \mathrm{~m} / \mathrm{s}, \rho_{1}=1.0$ et $\rho_{2}=4.5$,

* l'interface est située à $h=15 \mathrm{~mm}$, la surface émettrice à $z_{0}=20 \mathrm{~mm}$,

* la surface émettrice est carrée, son centre étant à la verticale du point focal,

* le champ reconstruit est calculé autour du point focal, parallèlement à l'interface,

\section{1) Comparaison entre les deux méthodes}

La figure 4 représente, pour une surface émettrice de côté $80 \mathrm{~mm}$, le champ de pression synthétisé par retournement temporel (courbe continue) et par la méthode de Fermat (courbe discontinue). On observe que la méthode de Fermat crée un lobe principal légèrement plus étroit que le retournement temporel, mais avec un niveau de lobes secondaires beaucoup plus élevé. En raison de la diffraction, le champ mesuré (phase 1 du retournement temporel) est maximal au centre du miroir, de sorte que les points du bord du miroir émettent moins que les points du centre au cours de la phase 2. Par contre, dans la méthode de Fermat, tous les points émettent avec une même force, ce qui explique le renforcement des lobes secondaires. Ce résultat est important car il permet d'apprécier le gain de focalisation résultant du retournement temporel, comparé à la méthode des surfaces de Fermat. On retrouve par ailleurs un résultat classique des transducteurs en surfaces de Fermat : ils présentent en général un niveau de lobes secondaires assez fort.

\section{2) Effet de la taille du miroir à retournement temporel}

De façon évidente, on peut s'attendre à ce que la qualité de refocalisation par retournement temporel soit d'autant meilleure que le miroir est plus grand. Afin de mettre cet effet en évidence, nous avons fait le même calcul avec deux miroirs de $80 \mathrm{~mm}$ et $20 \mathrm{~mm}$ de côté, le résultat étant représenté sur la figure 5. Cette figure montre clairement que la taille du miroir joue un rôle majeur, tant sur la taille du lobe principal que sur le niveau des lobes secondaires.

\section{4) Conclusion}

Dans cet article, nous avons présenté une comparaison entre l'approche du retournement temporel et la méthode des surfaces de Fermat pour optimiser la focalisation d'un champ ultrasonore à travers une interface plane séparant deux fluides homogènes. En régime monochromatique, la méthode des surfaces de Fermat semble légèrement meilleure que le retournement temporel pour le lobe principal (quoique cet effet a tendance à disparaître en régime transitoire), mais elle génère un niveau de lobes secondaires très élevé, ce qui peut être gênant, en particulier pour les applications d'imagerie. D'autres travaux sur des géométries d'interfaces plus complexes, sur des interfaces séparant fluides et solides, sont en cours pour compléter cette description du problème général de la focalisation dans les milieux stratifiés.

\section{5) Références}

[1] M. Fink, C. Prada, F. Wu et D. Cassereau, "Self-focusing in inhomogeneous media with timereversal acoustic mirrors", IEEE Ultrasonics Symposium, 1989, pp. 681-686,

[2] D. Cassereau, F. Wu et M. Fink, "Limits of self-focusing using closed time-reversal cavities and mirrors - Theory and experiment", IEEE Ultrasonics Symposium, 1990, pp. 1613-1618,

[3] M. Fink, IEEE Trans. Ultrason. Ferroelec. Freq. Contr. 39, 1992, pp. 555-566,

[4] F. Wu, J.L. Thomas et M. Fink, IEEE Trans. Ultrason. Ferroelec. Freq. Contr. 39, 1992, pp. $567-578$,

[5] D. Cassereau et M. Fink, IEEE Trans. Ultrason. Ferroelec. Freq. Contr. 39, 1992, pp. 579-592,

[6] D. Cassereau et D. Guyomar, J. Acoust. Soc. Am. 92, 1992, pp. 1706-1720,

[7] D. Cassereau et D. Guyomar, "Etude du champ impulsionnel réfléchi par un dioptre plan", Premier Congrès Français d'Acoustique, 1990, pp. 949-952.

[8] D. Cassereau et M. Fink, J. Acoust. Soc. Am. 94, 1993, pp. 2373-2386.

[9] G. R. Harris, J. Acoust. Soc. Am. 70, 1981, pp. 10-20, 


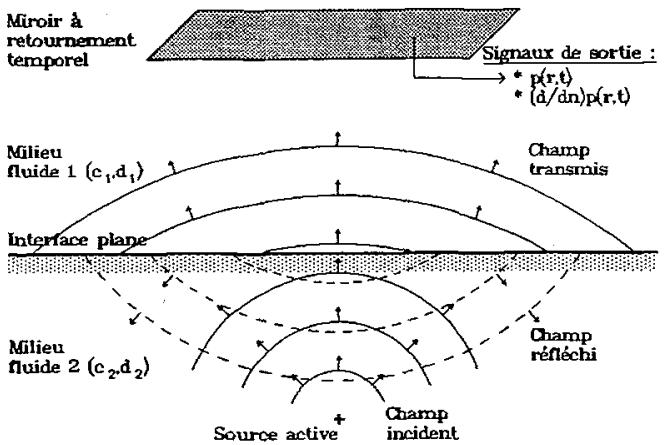

Figure 1 : Première étape du processus de retournement temporel : la source émet, le miroir écoute le champ transmis.

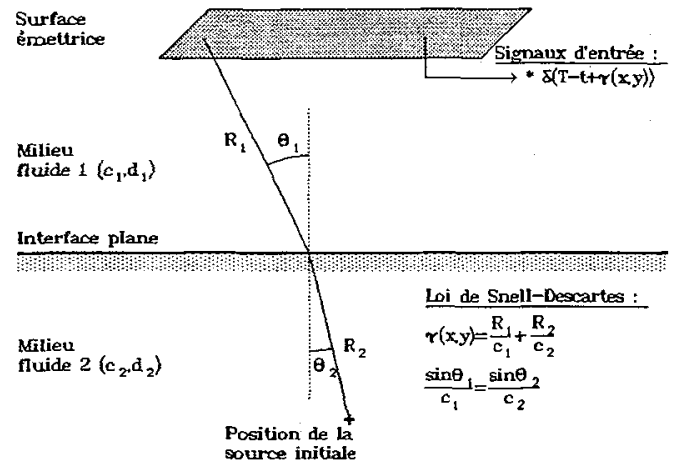

Figure 3 : Surface de Fermat : chacue point émet avec une même amplitude et un retard calculé suivant la loi de Snell-Descartes.

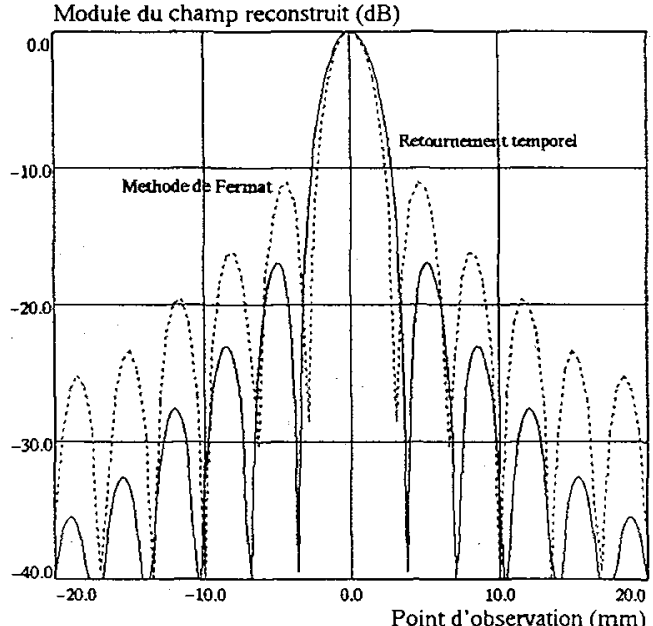

Figure 4 : Champ de pression synthétisé par retournement temporel (courbe continue) et par méthode de Fermat (courbe discontinue).

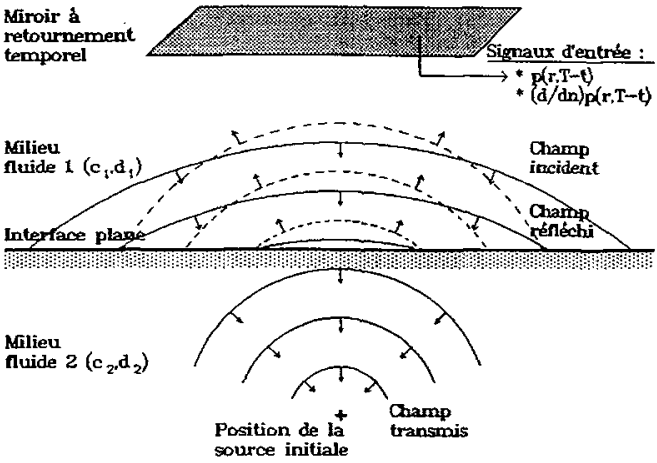

Figure 2 : Seconde étape du processus : le miroir réémet le retourné temporel de ce qu'il a mesuré lors de la première étape.

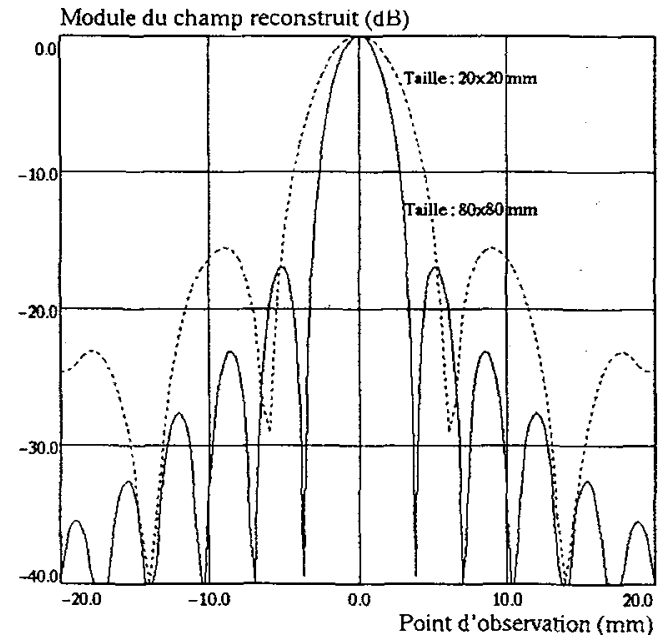

Figure 5 : Champ synthétisé par retournement temporel avec un miroir carré de côté $80 \mathrm{~mm}$ (courbe continue) et $20 \mathrm{~mm}$ (courbe discontinue). 